\title{
NEUTROPHIL GRANULOCYTES IN ATLANTIC SALMON (SALMO SALAR L.) ARE MHCII POSITIVE ANTIGEN-PRESENTING CELLS
}

\author{
Anita Rønneseth ${ }^{\S}$, Gyri T. Haugland, Lene Gundersen, Harald S. Lunde, Kaja Nordland and \\ Heidrun I. Wergeland
}

Department of Biology, University of Bergen, Norway

\begin{abstract}
In fish, like in mammals, professional antigen presenting cells like dendritic cells, macrophages and B cells are phagocytic cells that ingest, process and present antigens for T cells via MHCII. Neutrophils are professional phagocytic cells with an oxygen-dependent degradation mechanism. By use of a monoclonal antibody against neutrophils in Atlantic salmon, we have shown that the neutrophil granulocytes are MHCII-positive cells. The neutrophils were isolated by magnetic cell sorting and morphological characterization and phenotypic analyses were performed. After in vitro challenge with bacteria, IL12p40, which is essential for proliferation of naïve T cells, were upregulated. Further, Atlantic salmon was bath challenge with the same bacteria as the in vitro challenge experiment and the fraction of neutrophil granulocytes, B cells and MHCII positive cells in primary and secondary immune organs were measured by flow cytometry. Antigen-presentation in fish will be discussed.
\end{abstract}

\section{KEYWORDS}

Neutrophils, antigen-presenting cells, bacteria challenge, MHCII

$\S$ Corresponding author. Tel.: +47 55584421

E-mail address: Anita.Ronneseth@uib.no 